\title{
PICONE-TYPE THEOREMS FOR SEMIDISCRETE HYPERBOLIC EQUATIONS
}

\author{
KURT KREITH ${ }^{1}$ \\ Abstract. A comparison theorem of Picone-type is established for hyperbolic \\ boundary value problems by means of a semidiscrete approximation.
}

For ordinary and elliptic partial differential equations the familiar Sturm comparison theorem is readily extended to the more general Sturm-Picone Theorem [5]. However, in the case of hyperbolic partial differential equations a corresponding generalization poses significant difficulties. A likely explanation for these difficulties is that the usual proof of the Sturm comparison theorem is essentially an adjoint calculation [2] while the various proofs of the Sturm-Picone Theorem make use of tools from the calculus of variations. It is the lack of such variational tools in the hyperbolic case which precludes a direct generalization of familiar techniques.

On the other hand, there is strong physical motivation to suggest that Picone-type theorems should exist in the hyperbolic case. The equation

$$
\left(m(x, t) u_{t}\right)_{t}-\left(a(x, t) u_{x}\right)_{x}+p(x, t) u=0
$$

represents the $u$-displacement of a string of density $m(x, t)$ and tension $a(x, t)$, subject to a linear restoring force $p(x, t) u$. Given a second string

$$
\left(M(x, t) U_{t}\right)_{t}-\left(A(x, t) U_{x}\right)_{x}+P(x, t) U=0
$$

which is subject to a significantly larger restoring force (i.e. with $P \gg p$ ), it is reasonable to expect that (2) will, in some sense, oscillate faster than (1).

The case where $M \equiv m$ and $A \equiv a$ corresponds to the Sturm comparison theorem and has been dealt with by several authors $[2,4,6]$. A Picone-type theorem has been established in [3] in case $M$ and $m$ are independent of $x$ while $A$ and $a$ are independent of $t$. These assumptions allow for a generalized Liouville transformation which reduces the problem to the case $A \equiv a$ and $M \equiv m$.

The purpose of this note is to present another technique which applies in a more general setting. While we assume that $0<M(x, t) \equiv m(x, t)$, we shall allow for a general $A(x, t) \neq a(x, t)$. (In case $M$ and $m$ are independent of $x$, the change of variables $\tau=M t / m$ can always be used to achieve the equality $M \equiv m$.) Our

Received by the editors August 31, 1982.

1980 Mathematics Subject Classification. Primary 35B05.

Key words and phrases. Hyperbolic equations, Sturm comparison theorem, Picone theorem, semidiscrete system.

${ }^{1}$ Research supported by NSF MCS 8002130 
principal tool is a semidiscrete approximation to (1) and (2) corresponding to a system of discrete masses in place of a continuous string. The convergence of such semidiscrete approximations to the continuous solution is established in [1] and will not be considered here. However the (physically plausible) fact that such convergence takes place will be an implicit assumption in what follows. It is also assumed that all coefficients are continuous and that solutions are $C^{2}$ functions which satisfy the equaion in the classical sense.

THEOREM. Let $u(x, t)$ and $v(x, t)$ be nontrivial solutions, respectively, of

$$
\left(m(x, t) u_{t}\right)_{t}-\left(a(x, t) u_{x}\right)_{x}+p(x, t) u=0,
$$

and

$$
\left(m(x, t) v_{t}\right)_{t}-\left(A(x, t) v_{x}\right)_{x}+P(x, t) v=0,
$$

in a rectangular domain $D=\left\{(x, t): X_{1}<x<X_{2}, 0<t<T\right\}$. Suppose further that $u(x, t)$ is positive in $D$ and that $u$ and $v$ satisfy the boundary conditions

$$
\begin{aligned}
& u_{x}\left(X_{i}, t\right)+(-1)^{i} \sigma_{i} u\left(X_{i}, t\right)=0, i=1,2 ; 0 \leqslant t \leqslant T, \\
& u(x, 0)=u(x, T)=0, X_{1} \leqslant x \leqslant X_{2}, \\
& v_{x}\left(X_{i}, t\right)+(-1)^{i} \tau_{i} v\left(X_{i}, t\right)=0, \quad i=1,2 ; 0 \leqslant t \leqslant T .
\end{aligned}
$$

If

(i) $0 \leqslant \tau_{i}=\sigma_{i} \leqslant \infty$,

(ii) $a(x, t) \geqslant A(x, t)>0$ in $D$, and

(iii) $P(x, t)-p(x, t) \geqslant 2[a(x, t)-A(x, t)]$ in $D$.

Then $v(x, t)$ has a zero in $D$.

Proof. Replacing the continuous variable $x$ by discrete $x_{i}=X_{1}+(i / n)\left(X_{2}-X_{1}\right)$, $i=0, \ldots, n$, one obtains the semidiscrete systems

$$
\left(m_{i}(t) u_{i}^{\prime}\right)^{\prime}=-p_{i}(t) u_{i}+a_{i-1, i}(t)\left(u_{i-1}-u_{i}\right)+a_{i, i+1}(t)\left(u_{i+1}-u_{i}\right)
$$

and

$$
\left(m_{i}(t) v_{i}^{\prime}\right)^{\prime}=-P_{i}(t) v_{i}+A_{i-1, i}(t)\left(v_{i-1}-v_{i}\right)+A_{i, i+1}(t)\left(v_{i+1}-v_{i}\right),
$$

where $u_{0}=v_{0}=0=u_{n}=v_{n}$ and $1 \leqslant i \leqslant n-1$. Introducing the column vectors $\mathbf{u}=\operatorname{col}\left(u_{1}, \ldots, u_{n-1}\right)$ and $\mathbf{v}=\operatorname{col}\left(v_{1}, \ldots, v_{n-1}\right)$ we can rewrite $\left(3^{\prime}\right)$ and $\left(4^{\prime}\right)$ as matrix systems

$$
\left(M(t) \mathbf{u}^{\prime}\right)^{\prime}=g(t) \mathbf{u}
$$

and

$$
\left(M(t) \mathbf{v}^{\prime}\right)^{\prime}=G(t) \mathbf{v},
$$

where $M(t)=\operatorname{diag}\left(m_{1}(t), \ldots, m_{n-1}(t)\right)$ and $g(t)$ is a symmetric matrix with

$$
\begin{gathered}
g_{i i}=-p_{i}-a_{i-1, i}-a_{i, i+1}, \quad 1 \leqslant i \leqslant n-1, \\
g_{i, i+1}=g_{i+1, i}=a_{i, i+1}, \quad 1 \leqslant i \leqslant n-2, \\
g_{i j}=0 \quad \text { if }|i-j|>1 .
\end{gathered}
$$


The matrix $G(t)$ is analogously defined when lower case letters are replaced by capitals. Thus from $\left(3^{\prime \prime}\right)$ and $\left(4^{\prime \prime}\right)$ we readily obtain the identity

$$
p\left[\mathbf{v} \cdot M \mathbf{u}^{\prime}-\mathbf{u} \cdot M \mathbf{v}^{\prime}\right]^{\prime}=\mathbf{v} \cdot g \mathbf{u}-\mathbf{u} \cdot G \mathbf{v}=\mathbf{v} \cdot(g-G) \mathbf{u} .
$$

Suppose now, to the contrary, that (4) has a solution $v(x, t)$ which is positive in $D$. Then for sufficiently large $n, v_{i}(t)>0$ for all $i, 1 \leqslant i \leqslant n-1$. Integrating (5) from 0 to $T$, it follows from $u(x, 0)=u(x, T)=0$ that the left side of (5) yields a negative value. The desired contradiction is obtained by showing that

$$
\int_{0}^{T} \mathbf{v} \cdot(g-G) \mathbf{u} d t \geqslant 0
$$

Here hypothesis (ii) assures that the off diagonal terms $a_{i, i+1}-A_{i, i+1}$ of $g-G$ are nonnegative while hypotheses (i) and (iii) assure that $a_{0,1}=A_{0,1} ; a_{n-1, n}=A_{n-1, n}$ and that $P_{i}-p_{i} \geqslant a_{i-1, i}+a_{i, i+1}-A_{i-1, i}-A_{i, i+1}$ for $1 \leqslant i \leqslant n-1$. Thus the hypotheses of the theorem assure that the entries of $g-G$ are nonnegative, and this completes the proof.

REMARKS. (1) By way of application, it is natural to choose (3) in the form

$$
\left(m(t) u_{t}\right)_{t}-\left(a(x) u_{x}\right)_{x}+p(t) u=0
$$

so that a separation of variables yields rectangular nodal domains of the type required in the theorem. The strength of the theorem lies in the fact that equation (4) can be of quite general type.

(2) The hypothesis $a \geqslant A$ in the theorem reverses the corresponding hypothesis $A \geqslant a$ required in [3]. However any strict inequality $a>A$ must now be compensated for by a physically plausible requirement of the form $P \gg p$.

\section{REFERENCES}

1. G. A. Baker, Error estimates for finite element methods for second order hyperbolic equations, SIAM J. Numer. Anal. 13 (1976), 564-576.

2. K. Kreith, A Sturm theorem for partial differential equations of mixed type, Proc. Amer. Math. Soc. 81 (1981), 75-78.

3. __ Picone-type theorems for hyperbolic partial differential equations, Pacific J. Math. (to appear).

4. G. Pagan, An oscillation theorem for characteristic initial value problems in linear hyperbolic equations, Proc. Roy. Soc. Edinburgh Sect. A 77 (1977), 265-271.

5. C. A. Swanson, Comparison and oscillation theory of linear differential equations, Academic Press, New York, 1968. , A dichotomy of Sturmian theory, SIAM Rev. 20 (1978), 285-300.

Department of Mathematics, University of California, Davis, California 95616 